\title{
Identification of a novel cytosolic aldehyde dehydrogenase allele, ALDHIAI*4
}

\author{
Shelley M. Moore, ${ }^{*}$ Tiebing Liang, ${ }^{2}$ Tamara J. Graves, ${ }^{2}$ Kevin M. McCall, ${ }^{2}$ Lucinda G. Carr ${ }^{2}$ and Cindy L. Ehlers ${ }^{3}$ \\ 'Pharmacology Unit, Department of Paraclinical Sciences, Faculty of Medical Sciences, The University of the West Indies, \\ St. Augustine, Trinidad and Tobago \\ ${ }^{2}$ Indiana University School of Medicine, 975 W. Walnut Street, IB424, Indianapolis, IN 46202-5121, USA \\ ${ }^{3}$ Department of Molecular and Integrative Neurosciences, The Scripps Research Institute, 10550 N. Torrey Pines Rd, SP30-1501, \\ La Jolla, CA 92037, USA \\ *Correspondence to: Tel: +1 868663 8613; E-mail: smmatrix2k1@yahoo.com
}

Date received (in revised form): 24th February 2009

\begin{abstract}
This paper reports the identification of a novel cytosolic aldehyde dehydrogenase I (ALDHIAI) allele. One hundred and sixty-two Indo-Trinidadian and 85 Afro-Trinidadian individuals were genotyped. A novel ALDHIAI allele, $A L D H I A I * 4$, was identified in an Indo-Trinidadian alcoholic with an $A$ inserted at position -554 relative to the translational start site, $+\mathrm{I}$. It was concluded that a wider cross-section of individuals needs to be evaluated in order to determine the representative frequency of the allele, and to see if it is associated with risk of alcoholism.
\end{abstract}

Keywords: ALDHIAI, base pair, polymorphism, Trinidad and Tobago

\section{Introduction}

The human cytosolic enzyme aldehyde dehydrogenase 1 (ALDH1A1) functions mainly in acetaldehyde and neurotransmitter metabolism. It is also reported to play a major role in the production of retinoic acid, which is important for gene expression and tissue differentiation, and also in cyclophosphamide detoxification. ${ }^{1-3}$ It is found in various tissues, including the central nervous system (CNS), ${ }^{4}$ with highest levels in the liver. ${ }^{2}$ Research has implicated the enzyme in the development of alcohol dependence and other alcohol-use disorders, alcohol-induced flushing and sensitivity to alcohol. ${ }^{3,5,6}$

Normal ALDH1A1, is tetrameric and predominantly of cytosolic origin. This enzyme has a relatively high $\left.K_{\mathrm{m}}(50-100 \mu \mathrm{M})\right)^{7,8}$ which is far greater than recorded physiological concentrations
$(0.4-2.5 \mu \mathrm{M}) .^{9-12}$ In addition, this enzyme has low catalytic efficiency $\left(K_{\mathrm{cat}} / K_{\mathrm{m}}\right)^{13}$ for acetaldehyde metabolism and hence exhibits its importance in ethanol elimination. Research into ALDH1A1 kinetics with respect to various neurotransmitter aldehydes reveals a $K_{\mathrm{m}}$ of $2.4 \mu \mathrm{M}$ for 5 -hydroxyindole acetaldehyde and a $K_{\mathrm{m}}$ of $0.4 \mu \mathrm{M}$ and $1.5 \mu \mathrm{M}$ for 3,4-dihydroxyphenylacetaldehyde and phenylacetaldehyde, respectively, and near equivalent $K_{\text {cat }}$ values for these substrates. ${ }^{14}$ ALDH1A1 has also displayed a $K_{\mathrm{m}}$ of $0.06 \mu \mathrm{M}$ in retinaldehyde metabolism, with the $K_{\text {cat }}$ value equivalent to the value in acetaldehyde metabolism. ${ }^{8}$ These functions are so important that some researchers believe that this enzyme is essential for life, and this belief is supported by the evidence that no individual has yet been identified with a total absence of ALDH1A1 catalytic activity. ${ }^{15}$ 
The gene coding for this enzyme, ALDH1A1, encodes 501 amino acid residues and is located on human chromosome 9q21.13. Its transcript (NM_000698) contains 13 exons and the gene is approximately 52 kilobases $(\mathrm{kb})$ in length (NW_924484). ${ }^{16,17}$ In the promoter region, an ATA box and a CCAAT box are located 32 and 74 base pairs (bp), respectively, upstream from the transcription initiation site. The transcription initiation site is located $53 \mathrm{bp}$ upstream from the A of the initiation codon (ATG). ${ }^{18-20}$ Two polymorphisms have been previously identified in the promoter region of $A L D H 1 A 1$. The $A L D H 1 A 1 * 2$ allele contains a $17 \mathrm{bp}$ deletion from position -416 to -432 compared with the $A L D H 1 A 1 * 1$ allele, and the $A L D H 1 A 1^{*} 3$ allele has a three bp insertion at $-524 .^{3}$ ALDH1A1*2 was observed at frequencies of $0.035,0.023,0.023$ and 0.012 in Asian, Caucasian, Jewish and African-American individuals, respectively, while $A L D H 1 A 1^{*} 3$ was only observed in African-American individuals, at a frequency of $0.029 .^{3}$ These polymorphisms also have been observed in Mission Indians of Southwest California, where an allele frequency of 0.03 was detected for $A L D H 1 A 1 * 2$. Two subjects possessed the $A L D H 1 A 1^{*} 2$ allele and one subject displayed both the $A L D H 1 A 1^{*} 2$ and $A L D H 1 A 1^{*} 3$ alleles. $^{21}$

Trinidad and Tobago is a twin island country, located at the southern end of the Caribbean chain of islands, $10 \mathrm{~km}$ (seven miles) north-east of the coast of Venezuela. The population of the country is multi-ethnic but the two largest ethnic groups are those of East Indian $(40.0$ per cent, Indo-Trinidadians) and African (37.5 per cent, Afro-Trinidadians) descent. ${ }^{22}$ The ancestors of the Afro-Trinidadians were originally from West Africa and the Indo-Trinidadians came mainly from northern and southern India. The estimated rate of alcohol problems in this country is approximately 47 per cent for Indo-Trinidadians and 33 per cent for Afro-Trinidadians. ${ }^{23}$ The frequency of genotypes of alcohol metabolism in this population was unknown until recently. A study was undertaken that evaluated associations of $A L D H 1 A 1$ promoter polymorphisms with alcohol-related phenotypes in this population. ${ }^{24}$ In that study, the allele frequencies for $A L D H 1 A 1^{*} 1, A L D H 1 A 1^{*} 2$ and $A L D H 1 A 1 * 3$ in Afro-Trinidadians were found to be $0.941,0.035$ and 0.024 , respectively, and 0.926 , 0.074 and 0.000 in Indo-Trinidadians. The present paper reports the sequence of a novel allele identified in that study.

\section{Materials and methods}

\section{Subjects}

Patients were recruited from admissions to the substance abuse centres at Caura, San Fernando General and Scarborough Regional hospitals. There were no differences in admission or treatment based on ethnicity. Control subjects of both ethnic groups were matched by age, sex and ethnicity to the alcohol-dependent participants, and were recruited through fliers distributed in the communities and also by word of mouth. Whole-blood samples for genotyping were taken from a total of 247 individuals (162 IndoTrinidadian and 85 Afro-Trinidadian individuals), which included both alcohol dependent $(n=139)$ and non-alcohol-dependent $(n=108)$ subjects. Diagnosis of alcohol dependence was assessed using the Semi-Structured Assessment for the Genetics of Alcoholism (SSAGA). ${ }^{25,26}$ The study was carried out in accordance with the Declaration of Helsinki (2000) of the World Medical Association, and approval for the study was obtained from the ethics committees of the participating hospitals (San Fernando General, Caura and Scarborough Regional), the Faculty of Medical Sciences at the University of the West Indies and the Institutional Review Board (IRB) at The Scripps Research Institute. Informed, written consent was obtained from all participants before inclusion into the study.

\section{Genotyping}

Genomic DNA was isolated from dried blood spots. ${ }^{27}$ The primers, ALDH1A-forward $\left(5^{\prime}\right.$-GCACTGAAAATACACAAGACTGAT- $\left.3^{\prime}\right)$ and ALDH1A-reverse (5'-AGAATTTGAGGATTG AAAAGAGTC- $3^{\prime}$ ), were designed on the basis of human $A L D H 1 A 1$ exon 1 and promoter sequences 
(accession number M31982), and used in polymerase chain reaction (PCR) reactions to obtain $\left[\alpha-{ }^{33} \mathrm{P}\right]$ deoxycytidine triphosphate-radiolabelled fragments. Products were electrophoresed on 6 per cent acrylamide denaturing gels and scored on the basis of the mobility of each resulting PCR fragment.

\section{Results and discussion}

PCR analysis, using the ALDH1A1 forward and reverse PCR primers, generated products with corresponding sizes as follows: $A L D H 1 A 1 * 1=209 \mathrm{bp}$; ALDH1A1*2 $=192 \mathrm{bp} ; \quad$ ALDH1A1*3 $=212 \mathrm{bp}$ and $A L D H 1 A 1 * 4=210 \mathrm{bp}$ (Figure 1). These genetic variations have been previously detected in other populations. The $A L D H 1 A 1 * 2$ allele has been identified in diverse ethnic populations, including, Asians, Caucasians and African-Americans, while $A L D H 1 A 1 * 3$ has only been discovered thus far in African-Americans, Mission Indians and Afro-Trinidadians. ${ }^{3,21,24}$

In our sample, for one individual, an IndoTrinidadian alcohol-dependent subject, a slightly

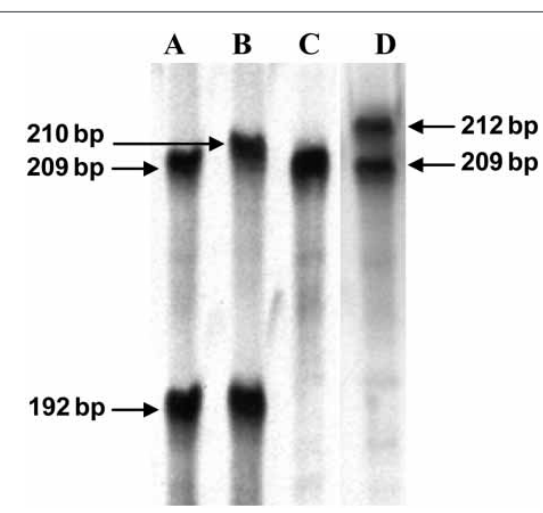

Figure I. Autoradiogram of four human ALDHIAI alleles and genotypes. PCR was used to generate $\left[\alpha-{ }^{33} \mathrm{P}\right]$ deoxycytidine triphosphate-radiolabelled fragments, which were separated by size on a 6 per cent acrylamide gel by electrophoresis. ALDHIAI*I is $209 \mathrm{bp}, A L D H I A I * 2$ I92 bp, ALDHIAI*3 2 I 2 bp and ALDHIAI*4 210 bp in length. The ALDHIAI*4 allele is a new finding reported here. The inserted nucleotide was confirmed by sequence analysis. (a) A heterozygous genotype ALDHIAI*I/*2. (b) A heterozygous genotype ALDHIAI*2/*4. (c) A homozygous genotype ALDHIAI*I/*I. (d) A heterozygous genotype ALDHIAI*I/*3. different allele size was detected on the autoradiogram; it appeared to be slightly larger than the ALDH1A1*1 allele and smaller than the $A L D H 1 A 1 * 3$ allele (Figure 1). By sequencing the PCR product, this unique allele was confirmed to have an $\mathrm{A}$ insertion at position -554 relative to the transcriptional start site (M31982). This new allele was named $A L D H 1 A 1^{*} 4$, in accordance with the nomenclature rules, and, therefore, the genotype of the subject would be $A L D H 1 A 1 * 2 / * 4$. The relative positions of the three polymorphisms are shown in Figure 2.

This new $A L D H 1 A 1 * 4$ allele was not discovered in any of our Afro-Trinidadian subjects and its frequency in other populations is not known. Therefore, future research in relation to this allele would have to incorporate a wider cross-section of the population of Trinidad and Tobago in order to determine the representative frequency of this allele in the respective ethnic groups. In addition, further analyses would be required to determine the expression difference, if any, of the ALDH1A1*4 isozyme.

Establishing the function and kinetics of the ALDH1A1*4 isozyme will also be valuable for the future. Cloning the mRNA and expressing, as well as isolating, the protein for kinetic analysis would lead ultimately to determining the $\mathrm{K}_{\mathrm{m}}$. These data will definitely add to the body of knowledge of aldehyde dehydrogenase enzymes and their associated genetic influences. Differences in expression may produce altered acetaldehyde, neurotransmitter and retinoic acid metabolism, and have an impact

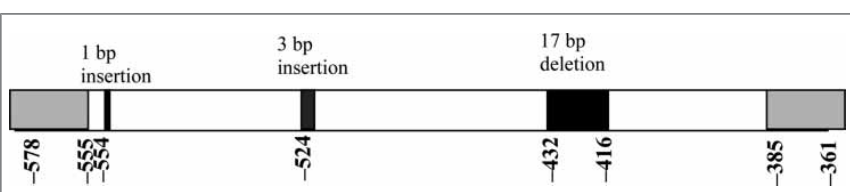

Figure 2. Schematic representation of the three human ALDHIAI polymorphisms. The region designates the fragment that was amplified for the genotyping assay from -36 I to -578 . The two grey boxes represent the forward and reverse primers. The relative positions of the three ALDHIAI polymorphisms are indicated with black boxes: the $17 \mathrm{bp}$ deletion (-416/-432), the 3 bp insertion (-524) and the I bp insertion (-554). The sequence flanking the inserted $A$ (in bold) is: ACTGATAACGATA. 
on the development of alcohol dependence and alcohol-related disorders, as well as on other physiological functions. Conducting similar studies in the populations of origin of our inhabitants (ie India and West Africa) could provide genotypic and phenotypic associations relating to the presence of these polymorphisms, and perhaps serve as predictors of alcohol disorders and other pathologies.

\section{Acknowledgments}

This research was supported, in part, by the National Institute of Alcoholism and Alcohol Abuse grants AA006420, AA014370, the Stein Endowment Fund (CLE), AA007611 (LGC) and a Dean's Award of the University of the West Indies, Trinidad and Tobago.

\section{References}

1. Yoshida, A., Rzhetsky, A., Hsu, L.C. and Chang, C. (1998), 'Human dehydrogenase gene family', Eur. J. Biochem. Vol. 251, pp. 549-557.

2. Vasiliou, V., Pappa, A. and Peterson, D.R. (2000), 'Role of aldehyde dehydrogenases in endogenous and xenobiotic metabolism', Chem. Biol. Interact. Vol. 129, pp. 1-19.

3. Spence, J.P., Liang, T., Eriksson, C.J.P., Taylor, R.E. et al. (2003), 'Evaluation of aldehyde dehydrogenase 1 promoter polymorphisms identified in human populations', Alcohol Clin. Exp. Res. Vol. 27, pp. 1389-1394.

4. Stewart, M.J., Malek, K., Xiao, Q., Dipple, K.M. and Crabb, D.W. (1995), 'The novel aldehyde dehydrogenase gene, ALDH5, encodes an active aldehyde dehydrogenase enzyme', Biochem. Biophys. Res. Commun. Vol. 211, pp. 144-151.

5. Ward, R.J., McPherson, A.J., Chow, C., Ealing, J. et al. (1994), 'Identification and characterisation of alcohol-induced flushing in Caucasian subjects', Alcohol Alcohol. Vol. 29, pp. 433-438.

6. Yoshida, A., Davé, V., Ward, R.J. and Peters, T.J. (1989), 'Cytosolic aldehyde dehydrogenase (ALDH1) variants found in alcohol flushers', Ann. Hum. Genet. Vol. 53, pp. 1-7.

7. Yoshida, A. (1992), 'Molecular genetics of human aldehyde dehydrogenase', Pharmacogenetics Vol. 2, pp. 139-147.

8. Yoshida, A., Hsu, L.C. and Davé, V. (1992), 'Retinal oxidation activity and biological role of human cytosolic aldehyde dehydrogenase', Enzyme Vol. 46, pp. 239-244.

9. Lieber, C.S. (1988), 'Metabolic effects of acetaldehyde', Biochem. Soc. Trans. Vol. 16, pp. 241-247.

10. Hatake, K., Taniguchi, T., Ouchi, H., Sakaki, N. et al. (1990), 'Possible involvement of kinins in cardiovascular changes after alcohol intake', Pharmacol. Biochem. Behav. Vol. 35, pp. 437-442.
11. Inoue, K., Fukunaga, M., Kiriyama, T. and Komura, S. (1984), 'Accumulation of acetaldehyde in alcohol-sensitive Japanese: Relation to ethanol and acetaldehyde oxidizing capacity', Alcohol. Clin. Exp. Res. Vol. 8, pp. 319-322.

12. Harada, S., Agarwal, D.P. and Goedde, H.W. (1981), 'Aldehyde dehydrogenase deficiency as cause of facial flushing reaction to alcohol in Japanese', Lancet Vol. 2, p. 982.

13. Ramchandani, V.A., Bosron, W.F. and Li, T.K. (2001), 'Research advances in ethanol metabolism', Pathol. Biol. Vol. 49, pp. 676-682.

14. MacKerell, A.D. Jr, Blatter, E.E. and Pietruszko, R. (1986), 'Human aldehyde dehydrogenase: Kinetic identification of the isozyme for which biogenic aldehydes and acetaldehyde compete', Alcohol. Clin. Exp. Res. Vol. 10, pp. 266-270.

15. Sládek, N.E. (2003), 'Human aldehyde dehydrogenases: Potential, pathological, pharmacological and toxicological impact', J. Biochem. Mol. Toxicol. Vol. 17, pp. 7-23.

16. Hsu, L.C., Yoshida, A. and Mohandas, T. (1986), 'Chromosomal assignment of the genes for human aldehyde dehydrogenase-1 and aldehyde dehydrogenase-2', Am. J. Hum. Genet. Vol. 38, pp. 641-648.

17. Raghunathan, L., Hsu, L.C., Klisak, I., Sparkes, R.S. et al. (1988), 'Regional localization of the human genes for aldehyde dehydrogenase-1 and aldehyde dehydrogenase-2', Genomics Vol. 2, pp. 267-269.

18. Hsu, L.C., Chang, W.C. and Yoshida, A. (1989), 'Genomic structure of the human cytosolic aldehyde dehydrogenase gene', Genomics Vol. 5, pp. 857-865.

19. Yanagawa, Y., Chen, J.C., Hsu, L.C. and Yoshida, A. (1995), 'The transcriptional regulation of human aldehyde dehydrogenase I gene. The structural and functional analysis of the promoter', J. Biol. Chem. Vol. 270, pp. 17521-17527.

20. Godbout, R. and Monckton, E.A. (2001), 'Differential regulation of the aldehyde dehydrogenase 1 gene in embryonic chick retina and liver', J. Biol. Chem. Vol. 276, pp. 32896-32904.

21. Ehlers, C.L., Spence, J.P., Wall, T.L., Gilder, D.A. et al. (2004), 'Association of ALDH1 promoter polymorphisms with alcohol-related phenotypes in Southwest California Indians', Alcohol. Clin. Exp. Res. Vol. 28, pp. 1481-1486.

22. Central Statistical Office (CSO) (2003), 'Population Census 2000', CSO; Port of Spain, Trinidad and Tobago.

23. National Alcohol and Drug Abuse Prevention Center (NADAPP) (2000), 'The Rapid Assessment Survey', NADAPP; Port of Spain, Trinidad and Tobago.

24. Moore, S., Montane-Jaime, K., Shafe, S., Joseph, R. et al. (2007), 'Association of ALDH1 promoter polymorphisms with alcohol-related phenotypes in Trinidad and Tobago', J. Stud. Alcohol Drugs Vol. 68, pp. 192-196.

25. Bucholz, K.K., Cadoret, R., Cloninger, C.R., Dinwiddie, S.H. et al. (1994), 'A new, semi-structured psychiatric interview for use in genetic linkage studies: A report on the reliability of the SSAGA', J. Stud. Alcohol Vol. 55, pp. 149-158.

26. Hesselbrock, M., Easton, C., Bucholz, K.K., Schuckit, M. et al. (1999), 'A validity study of the SSAGA: A comparison with the SCAN', Addiction Vol. 94, pp. 1361-1370.

27. Truett, G.E., Heeger, P., Mynatt, R.L., Truett, A.A. et al. (2000), 'Preparation of PCR-quality mouse genomic DNA with hot sodium hydroxide and tris (HotSHOT)', Biotechniques Vol. 29, pp. 52-54. 